Dubach, Josua; Schelleis, Natalie; Bölsterli, Katrin; Cacchione, Trix; Martarelli, Corinna S.; Probst, Matthias; Tempelmann, Sebastian

\title{
Unzugängliche Welten für das erfahrungsbasierte Lernen erschließen. Immersive Virtuelle Realität im naturwissenschaftlichen Sachunterricht
}

Sachunterricht in der Informationsgesellschaft. Bad Heilbrunn : Verlag Julius Klinkhardt 2022, S. 147-154. (Probleme und Perspektiven des Sachunterrichts; 32)

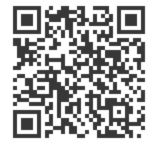

Quellenangabe/ Reference:

Dubach, Josua; Schelleis, Natalie; Bölsterli, Katrin; Cacchione, Trix; Martarelli, Corinna S.; Probst, Matthias; Tempelmann, Sebastian: Unzugängliche Welten für das erfahrungsbasierte Lernen erschließen. Immersive Virtuelle Realität im naturwissenschaftlichen Sachunterricht - In:

Sachunterricht in der Informationsgesellschaft. Bad Heilbrunn : Verlag Julius Klinkhardt 2022, S. 147-154 - URN: urn:nbn:de:0111-pedocs-242113 - DOI: 10.25656/01:24211

https://nbn-resolving.org/urn:nbn:de:0111-pedocs-242113

https://doi.org/10.25656/01:24211

in Kooperation mit / in cooperation with:

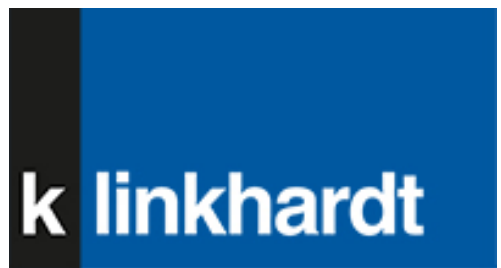

http://www.klinkhardt.de

\section{Nutzungsbedingungen}

Dieses Dokument steht unter folgender Creative Commons-Lizenz: http://creativecommons.org/licenses/by-nc-sa/4.0/deed.de - Sie dürfen das Werk bzw. den Inhalt unter folgenden Bedingungen vervielfältigen, verbreiten und öffentlich zugänglich machen sowie Abwandlungen und Bearbeitungen des Werkes bzw. Inhaltes anfertigen: Sie müssen den Namen des Autors/Rechteinhabers in der von ihm festgelegten Weise nennen. Dieses Werk bzw. der Inhalt darf nicht für kommerzielle Zwecke verwendet werden. Die neu entstandenen Werke bzw. Inhalte dürfen nur unter Verwendung von Lizenzbedingungen weitergegeben werden, die mit denen dieses Lizenzvertrages identisch oder vergleichbar sind.

Mit der Verwendung dieses Dokuments erkennen Sie die Nutzungsbedingungen an.

\section{Terms of use}

This document is published under following Creative Commons-License: http://creativecommons.org/licenses/by-nc-sa/4.0/deed.en - You may copy, distribute and transmit, adapt or exhibit the work in the public and alter, transform or change this work as long as you attribute the work in the manner specified by the author or licensor. You are not allowed to make commercial use of the work. If you alter, transform, or change this work in any way, you may distribute the resulting work only under this or a comparable license.

By using this particular document, you accept the above-stated conditions of use.

\section{Kontakt / Contact:}

\section{peDOCs}

DIPF | Leibniz-Institut für Bildungsforschung und Bildungsinformation Informationszentrum (IZ) Bildung

E-Mail: pedocs@dipf.de

Internet: www.pedocs.de

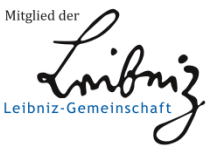




\section{Probleme und Perspektiven}

des Sachunterrichts

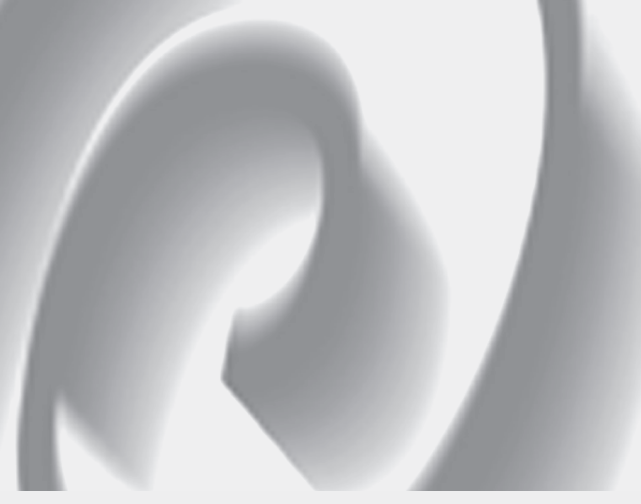

Andrea Becher / Eva Blumberg / Thomas Goll / Kerstin Michalik / Claudia Tenberge (Hrsg.)

Sachunterricht in der Informationsgesellschaft 
Probleme und Perspektiven

des Sachunterrichts

Band 32 


\section{Andrea Becher Eva Blumberg Thomas Goll \\ Kerstin Michalik Claudia Tenberge (Hrsg.)}

\section{Sachunterricht in der Informationsgesellschaft}


Schriftenreihe der

Gesellschaft für Didaktik des Sachunterrichts e.V.

Die Gesellschaft für Didaktik des Sachunterrichts (GDSU) e.V. ist ein Zusammenschluss von Lehrenden aus Hochschule, Lehrerfortbildung, Lehrerweiterbildung und Schule. Ihre Aufgabe ist die Förderung der Didaktik des Sachunterrichts als wissenschaftliche Disziplin in Forschung und Lehre sowie die Vertretung der Belange des Schulfaches Sachunterricht. www.gdsu.de

Bibliografische Information der Deutschen Nationalbibliothek Die Deutsche Nationalbibliothek verzeichnet diese Publikation in der Deutschen Nationalbibliografie; detaillierte bibliografische Daten sind im Internet abrufbar über http://dnb.d-nb.de.

2022.d. () by Julius Klinkhardt.

Druck und Bindung: Friedrich Pustet, Regensburg.

Printed in Germany 2022.

Gedruckt auf chlorfrei gebleichtem alterungsbeständigem Papier. 


\section{Inhaltsverzeichnis}

Andrea Becher, Eva Blumberg, Thomas Goll,

Kerstin Michalik und Claudia Tenberge

Editorial

Sachunterricht in der Informationsgesellschaft:

Sachunterrichtsdidaktische Rekonstruktion -

Medienbildung - informatische Bildung

Friedrich Gervé

Sachunterricht in der Informationsgesellschaft 17

Henrike Friedrichs-Liesenkötter

Die Ungleichheit und inklusive Medienbildung anhand

einer Betrachtung des Konnexes von Medien - Bildung - Flucht

Carsten Schulte

Digitale Technologien und informatische Bildung im Sachunterricht der Grundschule.

Sachunterricht in der Informationsgesellschaft:

Orte - Medien - Technologien

Michael Haider, Markus Peschel, Thomas Irion, Inga Gryl,

Daniela Schmeinck und Martin Brämer

Die Veränderung der Lebenswelt der Kinder und ihre Folgen

für Sachunterricht, Lehrkräftebildung und

sachunterrichtsdidaktische Forschung

Swaantje Brill und Alexandra Flügel

Digital unterwegs - außerschulische Lernorte

im Kontext digitaler Praktiken 
Svantje Schumann und Corinne Ruesch Schweizer

App-basierte Erfahrung und Reflexion als Unterstützung

der Professionalisierung von Lehrpersonen im Bereich BNE

Pascal Kihm und Markus Peschel

Gute Aufgaben 2.0 - Aufgaben und Aufgabenkulturen

im Rahmen der Digitalisierung

\section{Sachunterricht in der Informationsgesellschaft:}

Projekte im Kontext universitärer Lehrerbildung

Julia Peuke, Detlef Pech und Jara Urban

Zeitzeug*innengespräche und historisches Lernen

im Sachunterricht - ein Projektseminar.

Andreas Schmitt

Einfluss von Vorerfahrungen auf die

Fähigkeitsselbstkonzepte von Sachunterrichtsstudierenden

zu Kompetenzen in der digitalen Welt.

Friederike Kern, Volker Schwier und Björn Stövesand

Zum Ungleichgewicht digital vermittelten Sachunterrichts

und sprachlich-kommunikativer Anforderungen

Martin Brämer, Daniel Rehfeldt und Hilde Köster

Computational Thinking bei Sachunterrichtsstudierenden

im Lehr-Lern-Labor - Eine Rasch-Analyse

\section{Sachunterricht in der Informationsgesellschaft: \\ Perspektivenbezogene Zugangsweisen}

\section{Thomas Goll}

Political Literacy von Kindern -

Befunde, Implikationen, Herausforderungen

Melanie Haltenberger, Florian Böschl und Katharina Asen-Molz

Das Modell der Didaktischen Rekonstruktion als Kriterienraster

für studentische Erklärvideos nutzen - Ergebnisse aus einem standortübergreifenden Seminar zur geographischen Perspektive 
Josua Dubach, Natalie Schelleis, Katrin Bölsterli, Trix Cacchione,

Corinna S. Martarelli, Matthias Probst und Sebastian Tempelmann

Unzugängliche Welten für das erfahrungsbasierte Lernen erschließen:

Immersive Virtuelle Realität im naturwissenschaftlichen Sachunterricht........147

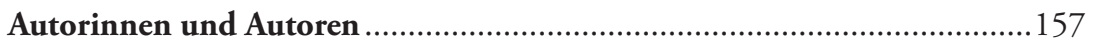


Josua Dubach ${ }^{1 *}$, Natalie Schelleis*, Katrin Bölsterli, Trix Cacchione, Corinna S. Martarelli, Matthias Probst und Sebastian Tempelmann

\section{Unzugängliche Welten für das erfahrungsbasierte Lernen erschließen: Immersive Virtuelle Realität im naturwissenschaftlichen Sachunterricht}

Active experience and manipulation are crucial sources of learning. However, many scientific topics are not accessible to our senses, such as microscopic or macroscopic structures and processes. By combining immersive and interactive elements, virtual reality offers a promising way to connect inaccessible parts of the world to direct sensory experience. This article explores possible guidelines for creating effective learning environments by reviewing opportunities and risks related to the use of virtual reality in science education. These ideas are illustrated with an example of virtual-reality-based teaching about the water cycle in primary school.

\section{Einleitung}

Das aktive Erleben und Manipulieren der Umwelt ist eine entscheidende Quelle des Lernens (z.B. Gopnik \& Wellman 2012). Die Bedeutung von sensorischen Erfahrungen und Handlungen für die Begriffsbildung wird sowohl von Entwicklungstheorien (Carey 2009; Piaget 1967) als auch von neurowissenschaftlichen und linguistischen Theorien der Begriffsbildung (Blouw, Solodkin, Thagard \& Eliasmith 2016) betont. Darüber hinaus wird die Relevanz der situierten Aktivität, durch die Wissen erworben wird, auch von vielen Bildungstheorien hervorgehoben (z.B. Collins, Brown, \& Newman 1988). Jedoch entziehen sich einige Aspekte der Umwelt unserer Wahrnehmung und sind für Handlungen nicht direkt zugänglich. Beispielsweise können weder Elemente und Strukturen des Mikrokosmos (z.B. Moleküle) oder des Makrokosmos (z.B. Sonnensystem) noch Prozesse, die sich über einen sehr kurzen oder einen sehr langen Zeitraum erstrecken, vollständig erfahren werden. Durch wissenschaftliche Techniken und

1 *geteilte Erstautor*innenschaft 
Theoriebildung können diese Aspekte zwar konzeptionell fassbar gemacht werden, das Wissen kann aber nicht oder nur sehr geringfügig in sensorischer Erfahrung verankert werden. Immersive Virtuelle Realität (IVR) hat das Potential, diese Verankerung herzustellen, indem beliebige virtuelle Welten dreidimensional und in $360^{\circ}$ aus der Ich-Perspektive erlebt werden. Um den Nutzen von IVR für den Sachunterricht zu beurteilen, ist eine Verzahnung von Entwicklungs- und Wirksamkeitsforschung erforderlich, wodurch komplexe interdisziplinäre Arbeitsabläufe entstehen.

Dieser Artikel geht der Frage nach, inwiefern IVR den Sachunterricht bereichern kann und gibt einen Einblick in mögliche Anwendungsfälle. Hierfür werden Chancen und Risiken beim Lernen mit IVR anhand von psychologischer und fachdidaktischer Fachliteratur beleuchtet. Daraus werden Arbeitshypothesen zur Gestaltung von IVR-Anwendungen für den Sachunterricht abgeleitet. Exemplarisch illustriert wird die Umsetzung durch eine Unterrichtseinheit zum Wasserkreislauf (WK) für 11- bis 12-jährige Kinder (5. und 6. Klasse). Eingebettet ist diese Entwicklung in ein interdisziplinäres Forschungsprojekt, welches vom Schweizerischer Nationalfonds im Rahmen des Nationalen Forschungsprogramms „Digitale Transformation“ gefördert wird. ${ }^{2}$

\section{Grundlagen}

IVR sind Computersysteme, die virtuelle Umwelten abhängig vom Nutzungsverhalten simulieren und sensorisch darstellen. Entscheidend ist die Verwendung eines Head-Mounted-Displays (VR-Brille), was dreidimensionales Sehen mit $360^{\circ}$-Umschau bewirkt. Dazu können weitere Schnittstellen kommen, bspw. über Hand-Tracking oder Controller, was das Interagieren mit den eigenen Händen erleichtert. Virtuelle Welten können auch auf PCs erlebt werden, jedoch ist die visuell-räumliche Immersion (fortan: Immersion) in das virtuelle Geschehen dann gering. Unterschieden wird deshalb immersive VR (IVR) von Desktop-VR (DVR) (z.B. Bailey \& Bailenson 2017). Die Immersion bewirkt ein starkes Erleben von räumlicher Präsenz (Gefühl des Dortseins), welches andere mediale Effekte verstärken kann (z.B. Hartmann, Wirth, Vorderer, Klimmt, Schramm \& Böcking 2015).

Lernen mit IVR. Das für IVR charakteristische Präsenzerleben geht häufig mit positiven emotionalen und motivationalen Effekten einher (Hartmann et al. 2015), was sich - auf unterschiedlichen Wegen - günstig auf Lernprozesse auswirken kann (Makransky \& Petersen 2020). Ein weiteres Potential könnte in der physischen

2 Die Entwicklung erfolgt gemeinsam mit der Firma Ateo (Zürich). 
Komponente von IVR liegen: Durch körperliche Aktivierung können Wissensstrukturen - im Sinne des Embodied Learning - leichter und tiefer angelegt werden (z.B. Skulmowski \& Xu 2021; Johnson-Glenberg 2018). Eine erste Meta-Analyse findet jedoch nur schwach-positive Effekte von IVR gegenüber anderen, weniger immersiven Medien (Wu, Yu \& Gu 2020). Ein Grund für das Ausbleiben von starken Lernvorteilen kann kognitive Beanspruchung (Cognitive Load) sein (z.B. Parong \& Mayer 2021). In IVR werden Nutzer*innen allein durch die freie $3 \mathrm{D}-360^{\circ}$ Umsicht bereits von vielen Reizen erreicht. Zusätzliche interaktive Elemente beanspruchen ebenfalls kognitive Ressourcen, da jede Handlung geplant, ausgeführt und evaluiert wird. Diese Effekte könnten die Vorteile durch positive Emotion, Motivation und Embodiment eindämmen.

Chancen und Risiken von IVR im Sachunterricht. Mit IVR lassen sich Exkursionen und Experimente unabhängig von Raum und Zeit durchführen - die Optionen scheinen zunächst sehr vielversprechend. Gerade das Interagieren mit der Umwelt mit den eigenen Händen ist für den Sachunterricht eine interessante Eigenschaft, insbesondere weil die Umwelt um sonst nicht zugängliche Artefakte erweitert werden kann (Lui, McEwen \& Mullally 2020). Jedoch bleibt der mögliche sensorische Erfahrungsraum dabei, trotz beachtlicher technischer Fortschritte, eingeschränkt und stets vordefiniert, was die möglichen didaktischen Anwendungen limitiert. Unterscheiden können wir zwischen schwer zugänglichen Exkursionszielen, wie Vulkanen, der Tiefsee oder dem Weltraum (z.B. Stein 2021), die durch echte Daten generiert werden können und komplett unzugänglichen Zielen, deren sensorische Darstellungen auf (Denk-)Modellen beruhen. Gerade bei den unzugänglichen Zielen kann die Kombination aus Immersion und Interaktion Chance und Risiko zugleich sein: Irrtümliche Attributionen der Alltagswelt wie Animismen können typische Fehlvorstellungen des Sachunterrichts fördern (Coley \& Tanner 2015). Aufgrund des starken Präsenzerlebens könnte es für Kinder schwieriger sein, die dualen Repräsentationen zwischen Symbolen (den konkreten sensorischen Darstellungen) und Referenten (den theoretischen Vorstellungen) aufrecht zu erhalten (Bailey \& Bailenson 2017), d.h. Realität und Vorstellung können in gewissem Masse vermischt werden. Diese Gefahr ist beim Lernen mit Modellen bei Kindern gut dokumentiert (Heitzmann 2019). Obwohl einige beispielhafte IVR-Lehrmittel für unzugängliche Umweltaspekte entwickelt wurden und sich eine positive Wirkung andeutet (z.B. Lui et al. 2020), existiert bisher keine systematische Überblicksarbeit zu diesen spezifischen Anwendungsfällen von IVR, noch gibt es Studien zur experimentellen Manipulation einzelner Designelemente. 


\section{Prinzipien zur Gestaltung von IVR-Lehrmitteln im Sachunterricht}

Das im Folgenden beschriebene Set von Prinzipien leitet sich aus Theorien und Erkenntnissen unterschiedlicher Disziplinen ab. Es dient als Orientierungsrahmen unserer laufenden Forschung (vgl. Abschnitt 4) und hat keinen Anspruch auf Vollständigkeit. Es bezieht sich auf den spezifischen Anwendungsfall, bei dem IVR als didaktisches Medium verwendet wird, um sonst unzugängliche Aspekte der Umwelt virtuell erlebbar und damit auch der Interaktion zugänglich zu machen. Die Prinzipien beziehen sich dabei auf das gesamte Lernarrangement, welches aus unterrichtlicher Vor- und Nachbereitung und kurzen Arbeitsphasen mit IVR (IVR-Episoden) besteht ${ }^{3}$.

Forschend-entdeckendes Lernen ermöglichen. Das entdeckende Lernen lässt sich gemäß Hartinger und Lohrmann (2014) in drei Typen unterteilen: Endeckendes Lernen durch (i) Konfliktinduktion und Konfliktlösung, (ii) Beispiele und (iii) Experimentieren. Mit IVR erscheint eine Kombination aller genannten Grundformen erstrebenswert und möglich. Ein ergebnisoffenes freies Experimentieren ist in einer (vor-)programmierten IVR jedoch schwer umsetzbar. Konkret werden die Schüler*innen deshalb bei der Interaktion in und mit der virtuellen Realität im Sinne eines moderat konstruktivistischen Lernens durch Instruktionen begleitet (Einsiedler 2014) sowie die Reflektion darüber gezielt gefördert (Mayer 2004). Natur von (Denk-)Modellen beachten. Hier steht die Didaktik vor einem Dilemma: Die immersive Realität macht „real“, was nicht real ist und fördert so das Lernen über das Modell selbst, erschwert damit aber wahrscheinlich ein grundsätzliches Modellverständnis. Somit muss das virtuelle Erleben von Modellwelten von didaktischen Maßnahmen flankiert werden. Z.B. sollte das Vergleichen von Modelltypen oder das Lernen über den Zweck von Modellen in die Vor- und Nachbereitung einfließen. Konkret werden zudem zusätzliche Hinweise oder Strategien in die IVR-Episoden integriert.

Kognitive Beanspruchung minimieren. Sorgfältige Vorbereitung und gezieltes Setzen von Hinweisreizen kann helfen, die Wahrnehmung in der virtuellen Welt auf relevante Aspekte zu lenken. Die Informationsverarbeitungsprozesse in IVR-Episoden sollten sorgfältig auf ihre Notwendigkeit geprüft werden. Dies betrifft besonders den Realismus von Strukturen und Prozessen sowie den Grad an zugelassener Interaktivität (Skulmowski \& Xu 2021).

Embodiment ausschöpfen. Konkrete Stellschrauben hierfür befinden sich beim Input (Steuerung einer Handlung) und beim Output (Feedback auf eine Handlung). Seitens der Steuerung werden möglichst große und inhaltlich kongruente Gesten diskutiert (Johnson-Glenberg 2018). Beim Handlungsfeedback sollten

3 Hier kann aus Platzgründen nur eine kleine Auswahl präsentiert werden. 
kontingente, multimodale sensorische Rückmeldungen beim Lernen unterstützen. Diese sind nicht nur für das Erleben von Präsenz essentiell (Hartmann et al. 2015), sondern auch beim Lernen von Zusammenhängen und Kausalität in der menschlichen Entwicklung (Buehner 2017).

Positive Emotionen ermöglichen. Das Potential von IVR, Menschen emotional anzusprechen und zu begeistern sollte als Lernfaktor nicht ungenutzt bleiben. Hierfür kommen zahlreiche Stellschrauben infrage: Farben, Geräusche, Narrative mit interessanten Charakteren oder spielerische Interaktionen.

\section{Implementierung am Beispiel des Wasserkreislaufes}

Der Wasserkreislauf (WK) zeichnet sich durch eine Reihe von schwer zugänglichen oder unzugänglichen Strukturen und Prozessen aus: Verdunstung und Kondensation sind als Prozesse weitestgehend unsichtbar, Wolken schwer zu erreichen und die häufig mit dem Teilchenmodell erklärten mikrokosmischen Bereiche der sensorischen Erfahrung unzugänglich. Der WK ist im Zyklus 2 (d.h. die Klassenstufen 3 bis 6) ein zentrales Thema im Schweizer Lehrplan 21 (D-EDK 2016).

Ablauf der Unterrichtseinheit. Die Unterrichtseinheit beinhaltet sechs Doppellektionen, in welchen sich die Lernenden mit den Phänomenen und Prozessen des WK auseinandersetzen, welche v.a. den „kleinen WK“ betreffen, also Prozesse der Verdunstung, Kondensation und Wolkenbildung sowie der Entstehung von Regentropfen. Zentral für das Verständnis sind hierbei das Teilchenmodell und der Temperaturgradient der Atmosphäre 4 . Die Lerneinheit beginnt mit einer holistischen Betrachtung des WKs im Sinne eines Advanced Organizers, anschließend werden die Teilprozesse gesondert betrachtet und zum Schluss der Prozess wieder als Ganzes. Ein zentrales Element der Einheit ist das Entwickeln und Überprüfen von Hypothesen. Dabei werden die Kinder mit einem Phänomen wie beispielsweise der Verdunstung von Wasser in der realen Welt konfrontiert. Ausgehend von ihren Vorstellungen bilden sie Hypothesen für die Wirkungszusammenhänge und Ursachen. Diese Hypothesen können sie dann in der virtuellen Welt überprüfen, indem sie sich bspw. in die (Modell-)Welt der Teilchen teleportieren und den Prozess der Verdunstung auf Modellebene erleben können. Der Austausch über die gemachten Erfahrungen sowie die Diskussion der Hypothesen erfolgt im Schulzimmer.

Elemente der virtuellen Welt. Nach dem Aufsetzen der VR-Brille finden sich die Kinder in einer Landschaft mit See und Bergen wieder. Alle wesentlichen Struk-

4 Zudem werden horizontale Luftmassenverschiebungen, Prozesse der Versickerung, der Pflanzentranspiration und die Verdunstung in Abhängigkeit der Oberflächenstruktur thematisiert. 
turen und Prozesse sind moderat realistisch dargestellt. Zusätzlich enthält die virtuelle Welt fiktive Elemente. Dazu gehören ein Non-Player-Character (NPC), ein Roboter mit menschlichen Zügen, sowie eine kleine Forschungsplattform mit einem großzügigen Deck. Das Deck dient dabei als Menü: Je nach Lektion befinden sich dort nur jene Gegenstände, mit denen die Kinder im Augenblick interagieren können. Zusätzlich kanalisieren visuelle und akustische Hinweisreize die Aufmerksamkeit, damit wesentliche Änderungen in der Umgebung nicht verpasst werden. Weitere Orientierung bietet der NPC. Er strukturiert die Lernerfahrungen, indem er problemorientierte Instruktionen, Hinweise und Erklärungen gibt. Bei längeren Pausen oder missglückten Interaktionen hilft diese Figur ebenfalls weiter. Insgesamt wird durch das Verhalten und Narrativ des NPCs eine wechselseitige Lehr-Lern-Beziehung angestoßen.

Handeln in der virtuellen Welt. Um etwas über unzugängliche Strukturen und Prozesse des WKs zu erfahren, stehen den Kindern verschiedene Arten von Handlungen zur Verfügung: Sie können Einflussfaktoren (z.B. die Umgebungstemperatur) manipulieren oder Prozesse spielerisch imitieren; sie können zwischen Modell-, Mikro- und Mesoebene wechseln und z.B. Moleküle berühren und manipulieren. Konkret bedeutet dies beispielsweise, dass sie nicht nur den Prozess der Verdunstung durch Temperaturveränderung auslösen bzw. beschleunigen, sondern zusätzlich den Vorgang auf der Modellebene betrachten können. Neben visuellen und auditiven Reizen erleben die Kinder auch haptische Rückmeldungen (z.B. vibrieren Wassermoleküle je nach Umgebungstemperatur unterschiedlich stark). Die Handlungen mit möglichst kongruenten Gesten bzw. Instrumenten zu versehen, gestaltete sich als herausfordernd. Bei alltagsnäheren Interaktionen kann leichter auf bestehende Konzepte zurückgegriffen werden: Um die Umgebungstemperatur zu ändern, muss ein Regler mit einer Hand hoch (wärmer) oder runter (kälter) gefahren werden. Wie aber sollte ein Wechsel in die Teilchenebene initiiert werden? Entschieden haben wir uns für eine spezielle Lupe mit zwei Modi: Sie kann auf mikroskopische Ebene vergrößern und bspw. kleinste Tröpfchen der Wolken sichtbar machen, und sie kann Wassermoleküle der Modellwelt sichtbar machen. Um der Vorstellung eines Kontinuums entgegenzuwirken, müssen die Modi per Knopfdruck ein- oder ausgeschaltet werden, sodass die Wechsel dichotom und abrupt erscheinen. 


\section{Ausblick}

IVR eröffnet für den Sachunterricht vielversprechende innovative Perspektiven. Als innovativ kann etwas jedoch erst bezeichnet werden, wenn es sich umsetzen lässt und sich langfristig durchsetzen kann (Hauschildt, Salomo, Schulz \& Kock 2016). Dafür ist neben der Praktikabilität in einem Schulsetting in erster Linie die Lernwirksamkeit ausschlaggebend (Gervé 2015). Ziel des Forschungsprojekts ist es, beide Kriterien zu untersuchen. Die gesamte Unterrichtseinheit wird nun bezüglich ihrer Umsetzbarkeit in einer Schulsituation erprobt. In einem kontrollierten 2x2-Design werden auch die Einflüsse von immersiven und interaktiven Elementen auf das Lernen getestet. Konkret werden acht Klassen unterrichtet, wobei jeweils zwei Klassen die VR-Umgebung in einer bestimmten Bedingung erleben (DVR passiv/aktiv, IVR passiv/aktiv). Für die gezieltere Überprüfung der globalen Wirkungszusammenhänge werden zusätzlich kontrollierte Labortests durchgeführt. Die so gewonnenen Erkenntnisse führen im Sinne des Design-Based Research (z.B. Bakker 2019) zu einer Weiterentwicklung der Lernumgebung. Damit verbinden wir Grundlagenforschung mit exemplarischer Implementierungsforschung.

\section{Literatur}

Bailey, J. O. \& Bailenson, J. N. (2017): Immersive Virtual Reality and the Developing Child. In: Cognitive Development in Digital Contexts. Elsevier, 181-200.

Bakker, A. (2019): Design principles in design research: A commentary. In: Bikner-Ahsbahs, A. \& Peters, M. (Hrsg.): Unterrichtsentwicklung macht Schule. Wiesbaden, S. 177-192.

Blouw, P., Solodkin, E., Thagard, P. \& Eliasmith, C. (2016): Concepts as Semantic Pointers: A Framework and Computational Model. In: Cognitive Science, 40(5), 1128-1162.

Buehner, M. J. (2017): Space, Time, and Causality. The Oxford Handbook of Causal Reasoning. Oxford.

Carey, S. (2009): The origin of concepts. Oxford.

Coley, J. D. \& Tanner, K. (2015): Relations between Intuitive Biological Thinking and Biological Misconceptions in Biology Majors and Nonmajors. In: CBE—Life Sciences Education, 14(1), ar8. https://doi.org/10.1187/cbe.14-06-0094 [04.08.2021].

Collins, A., Brown, J. S. \& Newman, S. E. (1988): Cognitive Apprenticeship: Teaching the Craft of Reading, Writing and Mathematics. In: Thinking: The Journal of Philosophy for Children, 8(1), 2-10.

D-EDK. (2016): Lehrplan 21. Fachbereichslehrplan Natur, Mensch, Gesellschaft. https://v-fe.lehrplan.ch/container/V_FE_DE_Fachbereich_NMG.pdf [07.03.2021].

Einsiedler, W. (2014): Lehr-Lern-Konzepte für die Grundschule. In: Einsiedler, W., Götz, M., Hartinger, A., Heinzel, F., Kahlert, J. \& Sandfuchs, U. (Hrsg.): Handbuch Grundschulpädagogik und Grundschuldidaktik. 4. Aufl. Bad Heilbrunn, S. 355-364.

Gervé, F. (2015): Digitale Medien. In: Kahlert, J., Fölling-Albers, M., Götz, M., Hartinger, A., Miller, S. \& Wittkowske, S. (Hrsg.): Handbuch Didaktik des Sachunterrichts. 2. Aufl. Bad Heilbrunn, S. 496-500. 
Gopnik, A. \& Wellman, H. M. (2012): Reconstructing constructivism: Causal models, Bayesian learning mechanisms, and the theory theory. In: Psychological Bulletin, 138(6), S. 1085-1108. https:// doi.org/10.1037/a0028044 [04.01.2022].

Hartinger, A. \& Lohrmann, K. (2014): Entdeckendes Lernen. In Einsiedler, W., Götz, M., Hartinger, A., Heinzel, F., Kahlert, J. \& Sandfuchs, U. (Hrsg.): Handbuch Grundschulpädagogik und Grundschuldidaktik. 4. Aufl. Bad Heilbrunn, S. 385-389.

Hartmann, T., Wirth, W., Vorderer, P., Klimmt, C., Schramm, H. \& Böcking, S. (2015): Spatial presence theory: State of the art and challenges ahead. In: Lombard, M., Biocca, F., Freeman, J., IJsselsteijn, W. \& Schaevitz, R. (Hrsg.): Immersed in Media. Cham, Heidelberg, New York, Dordrecht, London, S. 115-135.

Hauschildt, J., Salomo, S., Schulz, C. \& Kock, A. (2016): Innovationsmanagment. München.

Heitzmann, A. (2019): Modelle verwenden. In: Labudde, P. \& Metzger, S. (Hrsg.): Fachdidaktik Naturwissenschaft: 1.-9. Schuljahr. 3. Aufl. Bern, S. 89-104.

Johnson-Glenberg, M. C. (2018): Immersive VR and Education: Embodied Design Principles That Include Gesture and Hand Controls. Frontiers in Robotics and AI, 5. https://doi.org/10.3389/fr obt.2018.00081 [10.08.2021].

Lui, M., McEwen, R. \& Mullally, M. (2020): Immersive virtual reality for supporting complex scientific knowledge: Augmenting our understanding with physiological monitoring. In: British Journal of Educational Technology, 51(6), 2181-2199.

Makransky, G. \& Petersen, G. (2020): The Cognitive Affective Model of Immersive Learning (CAMIL): A Theoretical Research-Based Model of Learning in Immersive Virtual Reality. In: Educational Psychology Review, 937-958.

Mayer, R. E. (2004). Should There Be a Three-Strikes Rule Against Pure Discovery Learning? In: American Psychologist, 59(1), 14-19.

Parong, J. \& Mayer, R. E. (2021): Cognitive and affective processes for learning science in immersive virtual reality. In: Journal of Computer Assisted Learning, 37(1), 226-241.

Piaget, J. (1967): Psychologie der Intelligenz. Zürich.

Skulmowski, A. \& Xu, K. M. (2021): Understanding Cognitive Load in Digital and Online Learning: A New Perspective on Extraneous Cognitive Load. In: Educational Psychology Review. https://doi. org/10.1007/s10648-021-09624-7 [13.09.2021].

Stein, C. (2021): Wassermangel in Kenia als Folge des Klimawandels. Mit Virtual Reality Massnahmen zur Wassergewinnung vor Ort entdecken. In: geographie heute, 42(354), 30-33.

Wu, B., Yu, X. \& Gu, X. (2020): Effectiveness of immersive virtual reality using head-mounted displays on learning performance: A meta-analysis. In: British Journal of Educational Technology, 51(6), 1991-2005. 